\title{
Aptidão cardiorrespiratória, perfil lipídico e metabólico em adolescentes obesos e não-obesos
}

\author{
Neiva LEITE* \\ Gerusa Eisfeld MILANO* \\ Fabrício CIESLAK ${ }^{*}$ \\ *Núcleo de Pesquisa \\ em Qualidade de Vida, \\ Universidade Federal \\ Joice Mara Facco STEFANELLO* \\ do Paraná.
}

\section{Resumo}

0 objetivo deste estudo foi investigar a relação entre o consumo máximo de oxigênio, perfil lipídico e metabólico em meninas e meninos obesos e não-obesos. Estudo transversal, descritivo e correlacional. A amostra foi composta por 91 obesos e 30 não-obesos, dos 10 aos 16 anos. Avaliou-se o índice de massa corporal (IMC) e a circunferência abdominal (CA). A aptidão cardiorrespiratória foi avaliada de forma direta através da análise do consumo máximo de oxigênio $\left(\mathrm{V}_{2} \mathrm{2max}_{\mathrm{max}}\right.$. Determinaram-se niveis de colesterol total (CT), lipoproteina de alta densidade (HDL-C), lipoproteína de baixa densidade (LDL-C), triglicérides (TG), glicemia e insulinemia após 12 horas de jejum. Analisaram-se os dados pelo teste "t" de student e correlação parcial controlada pela idade, com um nível de significância de $p<0.05$. 0 grupo não-obeso apresentou maiores valores de $V 0_{2 \max }$ e HDL-C do que os obesos $(p<0,001)$. 0 TG $(p<0,01)$ e insulinemia $(p<0,001)$ foram superiores nos obesos comparados aos não-obesos. Os valores de LDL$C, C T$ e glicemia não diferiram entre os grupos. Houve correlação do $\dot{V} 0_{2 \max } \operatorname{com}$ o IMC $(r=-0,540 ; p<$ $0,001)$, CA $(r=-0,535 ; p<0,001)$, a insulinemia $(r=-0,392 ; p<0,001)$, TG $(r=-0,277 ; p=0,005)$ e $\mathrm{HDL}-\mathrm{C}(r=0,299 ; p=0,002)$. Não houve correlações do $\dot{V} 0_{2 \max }$ com a $C T$, a fração LDL-C e a glicemia.0 menor $\mathrm{VO}_{2 \text { max }}$ correlacionou com maiores adiposidade, TG e insulinemia, bem como redução de HDL. 0 $\dot{V} \mathrm{O}_{2 \max }$ não correlacionou com o CT, LDL-C e glicemia, sugerindo a importância do controle genético sobre estas variáveis e um menor tempo de influência do sedentarismo na população infanto-juvenil.

UnITERMOS: Adiposidade; Aptidão cardiorrespiratória; Lipídios; Metabolismo.

\section{Introdução}

O sobrepeso e a obesidade aumentaram em todas as faixas etárias nos países desenvolvidos e em desenvolvimento (COUTINHO, 2006), sendo considerados uma epidemia global e um problema de saúde pública (Oliveira \& Fisberg, 2003). O excesso de peso e a distribuição da gordura corporal elevam a possibilidade de alterações no perfil metabólico e complicações cardiovasculares em idades precoces (Bouchard, Després \& Mauriège, 1993; Thomas, Cooper, Williams, Baker \& Davies, 2007), principalmente na presença da obesidade visceral (Lee, Bracha \& Arlanian 2006), fazendo com que as doenças cardiovasculares sejam as causas mais frequentes de morbidade e mortalidade na vida adulta (LAKKA, LAAKSONEN, LAKKA, MANNIKKO, Niskanen, Rauramaa \& Solonen, 2003).

No Brasil, a prevalência do excesso de peso em adolescentes aumentou $359 \%$ nos meninos e 105\% nas meninas, nos últimos 30 anos (POF.IBGE, 2006). O excesso de peso na população infantojuvenil é preocupante em decorrência de seus impactos psicossociais negativos, da associação com co-morbidades tais como: dislipidemias, diabetes tipo II, hipertensão, problemas ortopédicos, 
respiratórios e maiores chances de alguns tipos de câncer (Kiess, Galler, Reich, Muller Kapellen, Deutscher, Raile \& Kratzsch, 2001; Leite, Cieslak, Milano, Góes, Lopes, Bento, Prestes \& Radominski, 2009a; Martinez, Moreno, MARQUEZ-Lopes \& MARTí, 2002).

O aumento expressivo na prevalência da obesidade infanto-juvenil relaciona-se principalmente à mudança no estilo de vida dos jovens, associada com a ingestão de alimentos inadequados (MeHTA \& ChANG, 2008) e a redução no nível de atividade física (DeForChe, Lefevre, Bourdeaudhuij, Hills, Duquet \& BOUCKAERT, 2003; YANCEY, WOLD, MCCARTHY, Weber, Lee, Simon \& Fielding, 2004). Tais comportamentos, assumidos como consequências do mau uso da tecnologia na sociedade moderna, conduzem a hábitos inativos e provocam reduções do gasto calórico e menor nível de aptidão cardiorrespiratória (TÖRÖK, SZELÉNYL, PÓRZÁsz \& Molnár, 2001).

As dislipidemias são as co-morbidades cardiovasculares mais frequentes em crianças e adolescentes obesos, diagnosticadas em aproximadamente 40 a 60\% dos indivíduos (LimA, Arrais, Almeida, Souza \& Pedrosa, 2004). Possivelmente essas alterações estão relacionadas também à inatividade física (STENSEL, Lin, Ho \& Aw, 2001). Estudos têm demonstrado resultados conflitantes na avaliação das dislipidemias e aptidão cardiorrespiratória na população infanto-juvenil (Shaibi, Cruz, Ball, Weigenberg, Kobaissi, Salem \& Goran, 2005; Stensel et al., 2001). Algumas

\section{Materiais e métodos}

\section{Delineamento do estudo}

O presente estudo de desenho transversal e descritivo foi realizado no período de fevereiro de 2004 a novembro de 2007, com pacientes da Unidade de Endocrinologia Pediátrica do Hospital de Clinicas (HC) de Curitiba e estudantes de escolas da região central de Curitiba (PR), na faixa etária de 10 a 16 anos, de ambos os sexos.

A avaliação antropométrica e a coleta sanguínea foram realizadas no Ambulatório de Endocrinologia Pediátrica do Hospital de Clínicas (HC) da Universidade Federal do Paraná, do município de Curitiba (PR). A avaliação cardiorrespiratória foi realizada no Departamento de Educação Física da evidências científicas têm sugerido que a menor aptidão cardiorrespiratória (LOFTIN, SOTHEEN, TRODCLAIR, O'Hanlon, Miller \& Udall, 2001; MafFeis, Schena, Zaffanello, Zoccante, Schultz \& Pinelli, 1994; Zanconato, Baraldi, Santuz, Rigon, Vido, Dalt \& ZACCHELLO, 1989) e o aumento nas dislipidemias (LimA et al., 2004) estão diretamente relacionados com a obesidade. SHAIBI et al. (2005), avaliando 163 obesos, encontraram uma associação inversa entre o volume máximo de oxigênio $\left(\dot{V} \mathrm{O}_{2 \max }\right)$ e os fatores de risco para co-morbidade. Sugerindo que a inatividade física esteja relacionada a alteraçóes metabólicas na infância.

Entretanto, em um estudo conduzido com 20 crianças obesas e 20 não-obesas, STENSEL et al. (2001) não encontraram associação entre a baixa capacidade aeróbia e as elevadas concentraçōes lipídicas dos sujeitos investigados. Indicando que, ainda na infância, o menor nível de condicionamento cardiorrespiratório não acarrete em alteração lipídica, pelo pouco tempo de evidenciar a influência negativa do sedentarismo nesta faixa etária.

Os resultados dessas investigações demonstraram discordância e lacuna científica na avaliação do perfil lipídico e aptidão cardiorrespiratória em crianças e adolescentes obesos. Mais estudos são necessários para esclarecer se existe associação entre o consumo máximo de oxigênio e alteraçôes no perfil lipídico na população infanto-juvenil obesa. Assim, o objetivo deste estudo foi investigar a relação entre o consumo máximo de oxigênio, perfil lipídico e metabólico em meninas e meninos obesos e nãoobesos.
Universidade Federal do Paraná. Ambas as avaliaçôes foram realizadas em dias distintos.

O protocolo de pesquisa foi delineado conforme as diretrizes propostas na Resolução 196/96 do Conselho Nacional de Saúde sobre pesquisas envolvendo seres humanos, registrado sob o número CEP/HC: 765.184/2003-11.

\section{Sujeitos}

A amostra foi obtida por conveniência, levando em consideração a disponibilidade dos pacientes para participarem das avaliações, e o consentimento dos responsáveis pelos menores mediante da assinatura do termo de consentimento livre e 
esclarecido. Para compor a amostra foram selecionados 91 indivíduos obesos (36 meninos, 55 meninas) e 30 não-obesos (13 meninos, 17 meninas) com idade entre 10 a 16 anos.

\section{Material}

Para determinar a massa corporal foi utilizada uma balança antropométrica com precisão de $100 \mathrm{~g}$ e capacidade máxima para $150 \mathrm{~kg}$, e para a estatura, um estadiômetro com escala de $0,1 \mathrm{~cm}$. A partir dessas duas variáveis, calculou-se o índice de massa corporal (IMC). O grupo com excesso de peso foi definido utilizando os valores médios de IMC para idade e sexo, estabelecendo-se como ponto de corte o percentil > $90^{\circ}$. O grupo não-obeso encontrou-se entre $5^{\circ}$ e $85^{\circ}$ percentil, conforme valores disponibilizados pelo CDC (Kuczmarski, Ogden, Grummer-Strawn, Flegal, Guo, Wei, Mei, curtin, Roche \& Johnson, 2000), para cada sexo e faixa etária.

A CA foi medida em cm, com uma fita flexível e inextensível, com precisão de $0,1 \mathrm{~cm}$, aplicada acima da crista ilíaca, paralela ao solo. Para essa medida, o indivíduo deveria estar em pé, com o abdome relaxado, com os braços ao longo do corpo e os pés unidos. Consideraram-se os valores acima ou iguais ao $75^{\circ}$ percentil como limítrofes ou aumentados, para idade e sexo (Fernandez, Redden, Petrobelli $\&$ AlLison, 2004).

A avaliação clínica foi realizada por um profissional da área de pediatria para detecção de eventuais doenças cardiovasculares e para determinar o grau de maturação sexual (TANner, 1962). Foram excluídos do estudo todos os indivíduos que apresentaram contraindicações para a realização das avaliações e que não foram classificados como púberes.

As amostras sanguíneas para a avaliação da glicemia, da insulinemia, do colesterol total (CT), HDL-C, LDL-C e triglicérides (TG) foram coletadas no período da manhã, após 12 horas de jejum. As concentrações plasmáticas de CT, TG e de HDL-C foram determinadas em $\mathrm{mg} / \mathrm{dL}$, utilizando-se o teste colorimétrico enzimático (CHOD - PAP) (Laboratório Merck, Darmstadt, Alemanha; Laboratório Roche, Indianápolis, IN, EUA). O LDL-C colesterol foi calculado pela seguinte fórmula: $\mathrm{LDL}-\mathrm{C}=\mathrm{CT}$ (HDL-C + TG/5) (Sociedade Brasileira de Cardiologia, 2007). Consideraram-se como referência os valores para lipídios apresentados na I Diretriz de Prevenção da Aterosclerose na Infância e na Adolescência (Sociedade Brasileira DE Cardiologia, 2005).
Os níveis de glicemia foram determinados com o método enzimático (Glicose Oxidase - Labtest) e classificando os indivíduos conforme o EXPERT Commitee on The Diagnosis and Classification of Diabetes Mellitus (1997). A insulina foi dosada pela técnica de quimiluminescência por imunoensaio imunométrico em $\mathrm{u} / \mathrm{ml}$, em equipamento automatizado, utilizando como referência 11,9 $\mathrm{u} / \mathrm{ml}$.

Para a análise cardiorrespiratória mensuraram-se as variáveis respiratórias e metabólicas através das trocas gasosas com um sistema metabólico direto (Vista XT metabolic sistem, EUA) computadorizado (Intel 486, DX2, $66 \mathrm{mhz}$ ), o qual forneceu informaçōes acerca do consumo de oxigênio $\left(\dot{V} \mathrm{O}_{2}\right)$ e da produção de dióxido de carbono $\left(\vec{V} \mathrm{CO}_{2}\right)$ e permitiu calcular a razão de troca respiratória $\left(\mathrm{RER}=\dot{V} \mathrm{CO}_{2} / \dot{V} \mathrm{O}_{2}\right)$. Foi utilizado um conjunto de máscara, gorro e turbina com vedação da boca e nariz levando o ar expirado ao equipamento de análise de gases.

A FC (em bpm) foi mensurada continuamente durante a realização do teste, através da utilização de cardiofrequencímetro (marca Polar ${ }^{\circledR}$, modelo S625X, Kempele, Finlândia).

O protocolo utilizado foi o de Balke modificado por RoWLAND (1990), mantendo a velocidade fixa em 3,25 mph e inclinação de 6\%, com incremento de $2 \%$ a cada três minutos, até o esforço máximo. Considerou-se $\dot{V} \mathrm{O}_{2 \max }$ quando dois dos seguintes critérios foram observados: a) exaustão ou inabilidade para manter a velocidade requerida; b) RER > 1,0 ; c) FC > 190bpm.

A avaliação do limiar ventilatório foi realizada de forma cega, por um avaliador experiente, que não tinha conhecimento de algum de cada indivíduo analisado. O limiar ventilatório foi determinado pelo método do equivalente ventilatório, onde a intensidade do exercício físico na qual verifica-se a ocorrência do primeiro aumento no equivalente ventilatório do oxigênio $\left(\dot{V} \mathrm{E} / \dot{V} \mathrm{O}_{2}\right)$ sem um concomitante aumento no equivalente ventilatório do dióxido de carbono ( $\bar{V} \mathrm{E} /$ $\dot{V} \mathrm{O}_{2}$ ) (Caiozzo, Davis, Ellis, Azus, Vandagriff, Prietto \& McMaster, 1982).

\section{Tratamento estatístico}

Para a análise dos dados fez-se uso da estatística descritiva. A normalidade dos dados foi analisada pelo teste de Kolmogorov-Smirnov, os dados não apresentavam distribuição normal foram transformados para a função logarítmica natural $(\log N)$. Em seguida recorreu-se ao teste de Student, para comparação dos grupos, a análise de correlação 
parcial ajustada para a idade foi utilizada para verificar o grau da associação da aptidão cardiorrespiratória e variáveis antropométricas com os parâmetros lipídicos e metabólicos. As análises foram realizadas por meio do programa estatístico Statistica 6.0®, considerando um nível de significância de 5\% $(\mathrm{p}<0,05)$.

\section{Resultados}

As características antropométricas de ambos os grupos podem ser observadas na TABELA 1 . O grupo obeso apresentou maiores valores de peso, IMC e CA do que o não-obeso $(\mathrm{p}<0,001)$. A idade e a estatura não diferenciaram entre os grupos.

TABELA 1 - Valores antropométricos dos indivíduos obesos e não-obesos.

${ }^{*} p<0,05$ ${ }^{* *} p<0,001$
${ }^{*} p<0,05$ ${ }^{* *} p<0,001$

\begin{tabular}{|c|c|c|c|c|c|}
\hline \multirow{3}{*}{ Variáveis } & \multicolumn{4}{|c|}{ Grupos } & \multirow[b]{3}{*}{$\mathrm{p}$} \\
\hline & \multicolumn{2}{|c|}{ Obeso } & \multicolumn{2}{|c|}{ Não-obeso } & \\
\hline & Média & DP & Média & DP & \\
\hline Idade (anos) & 13,3 & $\pm 1,63$ & 13,84 & $\pm 1,67$ & NS \\
\hline Peso (kg) & $76,01^{* *}$ & $\pm 15,05$ & 49,02 & $\pm 8,98$ & $<0,001$ \\
\hline Est $(\mathrm{cm})$ & 1,59 & $\pm 0,06$ & 1,61 & $\pm 0,1$ & NS \\
\hline IMC $\left(\mathrm{kg} / \mathrm{m}^{2}\right)$ & $29,51^{* *}$ & $\pm 4,57$ & 18,77 & $\pm 1,79$ & $<0,001$ \\
\hline $\mathrm{CA}(\mathrm{cm})$ & $96,94^{* *}$ & $\pm 12,06$ & 67,79 & $\pm 5,74$ & $<0,001$ \\
\hline
\end{tabular}

Os valores de $\dot{V} \mathrm{O}_{2 \max }$ e os parâmetros laboratoriais basais de ambos os grupos podem ser observados na TABELA 2. O grupo não-obeso apresentou maiores valores de $\dot{V} \mathrm{O}_{2 \max }$ e HDL-C do que o grupo obeso ( $\mathrm{p}<$ $0,001)$. Os dados de TG $(\mathrm{p}<0.01)$ e insulina basal $(\mathrm{p}<$ 0,001 ) foram superiores nos obesos do que nos não-obesos.

TABELA 2 - Valores de consumo máximo de oxigênio e perfil lipídico dos indivíduos obesos e não-obesos.

\begin{tabular}{|c|c|c|c|c|c|}
\hline \multirow{3}{*}{ Variáveis } & \multicolumn{4}{|c|}{ Grupos } & \multirow{3}{*}{$\mathrm{p}$} \\
\hline & \multicolumn{2}{|c|}{ Obeso } & \multicolumn{2}{|c|}{ Não-obeso } & \\
\hline & Média & DP & Média & DP & \\
\hline $\mathrm{VO}_{2 \max }\left(\mathrm{ml} \cdot \mathrm{kg}^{-1} \cdot \mathrm{min}^{-1}\right)$ & 33,94 & $\pm 5,18$ & $45,48^{* *}$ & $\pm 7,10$ & $<0,001$ \\
\hline $\mathrm{CT}(\mathrm{mg} / \mathrm{dl})$ & 156,17 & $\pm 32,34$ & 158,34 & $\pm 27,68$ & NS \\
\hline HDL-C (mg/dl) & 43,09 & $\pm 5,99$ & $54^{* *}$ & $\pm 11,39$ & $<0,001$ \\
\hline LDL-C (mg/dl) & 89,06 & $\pm 27,23$ & 88,51 & $\pm 23,55$ & NS \\
\hline TG $(\mathrm{mg} / \mathrm{dl})$ & $126,96^{*}$ & \pm 100 & 79,37 & $\pm 31,50$ & $<0,001$ \\
\hline Glicemia (mg/dl) & 89,81 & $\pm 8,22$ & 92,20 & $\pm 5,18$ & NS \\
\hline Insulina $(\mu \mathrm{UI} / \mathrm{ml})$ & $19,19^{* *}$ & $\pm 8,67$ & 6,83 & $\pm 2,86$ & $<0,001$ \\
\hline
\end{tabular}

Foram encontradas correlações do $\dot{V} \mathrm{O}_{2 \max }$ com o IMC $(\mathrm{r}=-0,540 ; \mathrm{p}<0,001)$, a CA $(\mathrm{r}=-0,535 ; \mathrm{p}$ $<0,001)$, a insulina $(\mathrm{r}=-0,392 ; \mathrm{p}<0,001)$, o TG $(\mathrm{r}=-0,277 ; \mathrm{p}=0,005)$ e HDL $(\mathrm{r}=0,299 ; \mathrm{p}=$ 0,002). Não foram encontradas correlações do $\dot{V} \mathrm{O}_{2 \max }$ com a CT, a fração LDL-C e a glicemia.

Os valores de IMC apresentaram correlaçôes com a CA $(\mathrm{r}=0,961 ; \mathrm{p}<0,001)$, a insulina $(\mathrm{r}=$ $0,678 ; \mathrm{p}<0,001)$; o HDL-C $(\mathrm{r}=-0,381 ; \mathrm{p}<$
$0,001)$ e o TG $(r=0,222 ; \mathrm{p}=0,025)$. Não houve correlação entre o IMC com CT, LDL-C e a glicemia.

A CA apresentou a mesma tendência nas correlaçōes. Ou seja, encontrou-se correlação para esta variável com a insulinemia $(r=0,678 ; p<$ $0,001)$, o HDL-C $(r=-0,384 ; \mathrm{p}<0,001)$ e o TG $(r=0,236 ; p=0,017)$, mas não com o CT, o LDL$\mathrm{C}$ e a insulinemia. 
TABELA 3 - Correlações entre os valores de aptidão cardiorrespiratória, variáveis antropométricas e lipídicas.

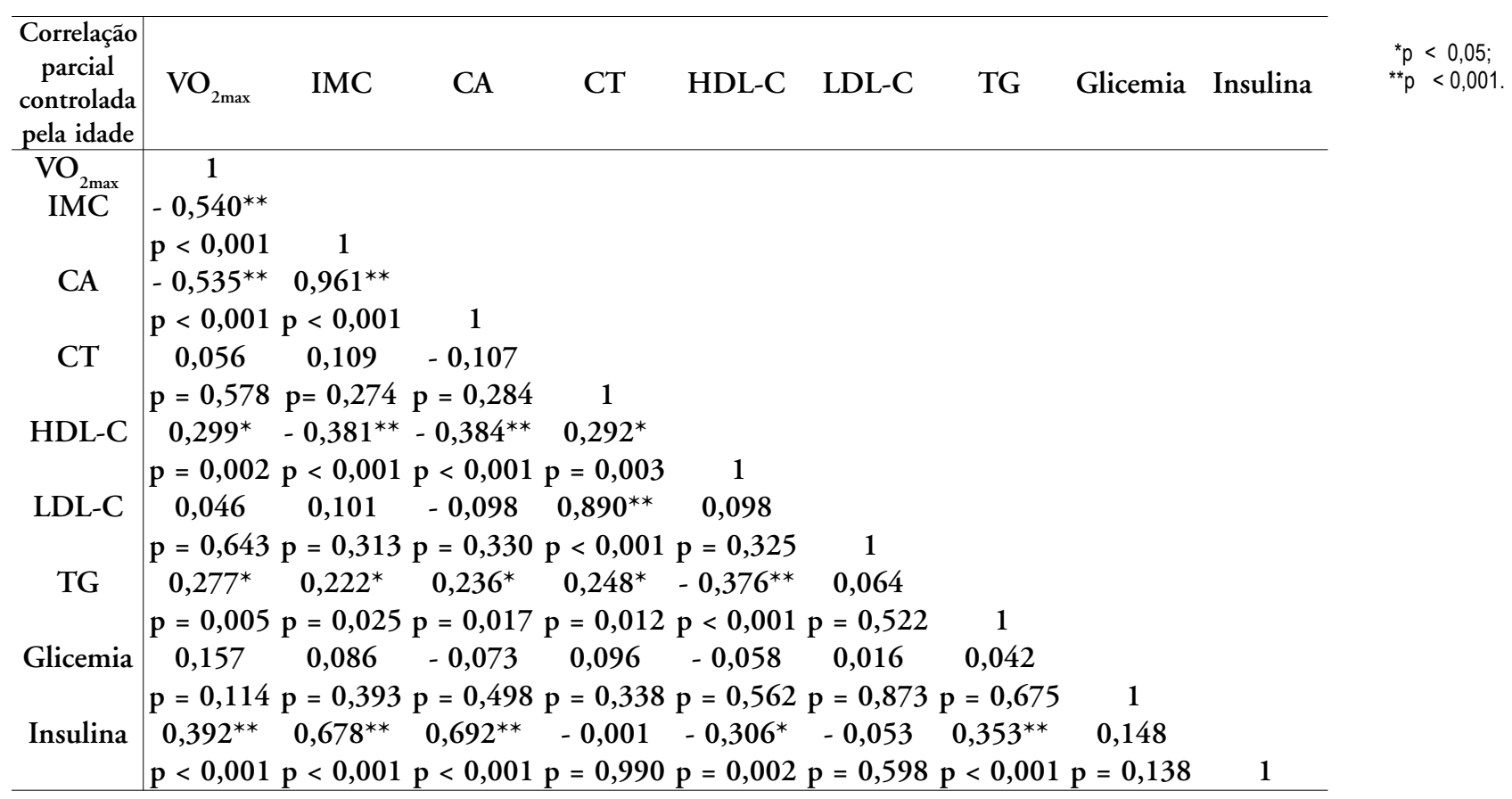

\section{Discussão}

A relação entre variáveis antropométricas, fatores de risco cardiovascular (CHU, RimM, WANG, LIOU \& ShieH, 1998; Freedman, Khan, Dietz, SRINIVASAN \& Berenson, 2001; Savva, Tornaritis, Savva, Kourides, Panagi, Silikiotou, Georgiou \& Kefatos, 2000) e aptidão cardiorrespiratória (SHAIBI et al., 2005; Thomas et al., 2007) em crianças têm sido o foco de algumas pesquisas. Em geral, os estudos relatam haver uma associação entre o perfil lipídico e obesidade (FreEdman et al., 2001; LeITE et al., 2009a; SAVVA et al., 2000), porém a relação com a aptidão aeróbia parece não estar muito clara (TÖRÖK et al., 2001). Nesta pesquisa, avaliaram-se as relações entre a obesidade, $o$ perfil lipídico e o consumo máximo de oxigênio em crianças e adolescentes obesos e não-obesos.

As medidas de IMC e CA são demonstradas como excelentes formas de avaliação da adiposidade global (LOHMAN, Roche \& MARTOREL, 1988) e central (Fernandez et al., 2004; LeE, Bracha \& Arlanian, 2006), sendo preditores independentes de DCV em crianças e adolescentes (FreEdman, Serdula, SRINIVASAN \& BERENSON, 1999; LeITE et al., 2009a). Alguns estudos nesta população encontraram que quanto mais elevados forem o IMC e a CA, maior é a probabilidade dos indivíduos apresentarem hipertensão arterial, hipertrigliceridemia, hipercolesterolemia e redução do HDL-C (FrEEDMAN et al., 2001; LISSAU, Overpeck, Ruan, Due, Holstein \& Hediger, 2004). Entretanto outros estudos não encontraram correlações entre a CA e os níveis de CT e de LDL-C, tanto em crianças (Guerra, DuARTE \& MOTA, 2001) e adolescentes (LEITE et al., 2009a), como em adultos, revelando a importância da influência genética sobre os níveis de CT e não somente o efeito negativo do sedentarismo, estilo de vida não saudável e adiposidade (Cercato, Mancini, Arguello, Passos, Villares \& HaLPERN, 2004).

$\mathrm{O}$ presente estudo não identificou correlação do IMC e da CA com os níveis de CT, LDL-C e glicemia. Por outro lado, houve correlação entre o IMC e a insulinemia, sugerindo que o aumento do IMC reflete diretamente no aumento da insulinemia (STENSEL et al., 2001). Os obesos apresentaram maiores valores de triglicerídeos e insulinemia em jejum do que os nãoobesos, sem diferenças no CT e LDL-C. O que contraria os achados no estudo realizado por BoYD, Koenigsberg, Falkner, Gidding e Hassink (2005), que avaliaram o perfil lipídico em 497 crianças entre dois à 18 anos com excesso de peso, os autores observaram que os adolescentes moderadamente obesos tiveram maiores proporções níveis de CT e LDL-C aumentado quando comparados com os severamente obesos. 
Por outro lado, os valores de HDL-C menores nos obesos do que nos não-obesos, encontrados no presente estudo, corroboram os resultados encontrados em outros estudos (BoyD et al., 2005; LeITE et al., 2009a), demonstrando que os adolescentes severamente obesos apresentaram diferenças estatísticas de HDL-C diminuído em relação aos moderadamente obesos. Além disso, o HDL-C demonstrou correlação com o IMC e a CA como verificada em outras evidências (Maffeis et al., 2001; LeE, BraCha \& ARLANian, 2006; LeITE et al., 2009a).

Nesta pesquisa encontrou-se relação dos níveis baixos de $\dot{V} \mathrm{O}_{2 \max }$ apenas com o TG, HDL-C e insulinemia. Porém, não houve correlações com o CT, a fração LDL-C e a glicemia. O estudo de STENSEL et al. (2001) encontrou associação entre o aumento do peso e alteraçōes lipídicas e também com os valores de insulinemia, porém não identificaram relação com a capacidade aeróbia. Da mesma forma, na pesquisa realizada por SHAiBi et al. (2005) não foram verificadas relaçôes das variáveis da síndrome metabólica com o $\dot{V} \mathrm{O}_{2 \max }$ em adolescentes nas análises de correlações parciais. Porém, a aptidão cardiorrespiratória em adolescentes com síndrome metabólica é reduzida (TöRÖK et al., 2001), e pode ser melhorada através da prática do exercício físico (Leite, Milano, CieslaK, LOPES, RODACKI, RADOMINSKI, 2009b).

Em pesquisas com adolescentes, os níveis de $\dot{V} \mathrm{O}_{2 \max }$ parecem ser independentes dos fatores lipídicos e metabólicos (SHAibi et al., 2005; StenSEL et al., 2001; THOMAS et al., 2007). Desse modo, sugere-se que o condicionamento cardiorrespiratório nesta faixa etária ainda não reflita alterações no perfil lipídico e metabólico. Entretanto, se não ocorrerem alterações no estilo de vida, possivelmente na fase adulta esses problemas poderão acarretar em maiores riscos de morbidade e mortalidade por doenças cardiovasculares (LAKKA et al., 2003).

Alguns fatores limitantes devem ser apontados, como ausência da avaliação de hábitos alimentares, consumo de álcool e tabagismo que podem ter interferido nas concentraçôes plasmáticas de lipídios. O delineamento dos participantes obtido por conveniência pode ter ocasionado um viés de seleção tanto em obesos como nos não obesos, entretanto se faz necessário pela dependência da autorização dos pais ou responsáveis. Sugere-se considerar essas limitações na comparação com os resultados obtidos em pesquisas futuras.

Conclui-se que houve inversa relação entre aptidão aeróbia e obesidade, TG e insulinemia, e direta com níveis de HDL-C. Porém, os níveis de CT, LDL-C e glicemia não se correlacionaram com a aptidão cardiorrespiratória na infância e adolescência, provavelmente associados ao pequeno tempo de exposição ao estilo de vida não saudável ou a presença de fatores genéticos influenciando o perfil lipídico. A relação entre o $\dot{V} \mathrm{O}_{2 \max }$ e as dislipidemias ainda não está muito clara, portanto evidencia-se a necessidade da realização de estudos prospectivos investigando os distúrbios do perfil lipídico na população infantojuvenil e a influência da prática de exercícios físicos.

\section{Abstract \\ Cardiorespiratory fitness, lipid and metabolic profile in obese and non-obese adolescents}

The aim this study was investigates the relationship between maximum oxygen consumption, lipid and metabolic profile in obese and non-obese girls and boys. Transversal, descriptive and correlational study. 91 obese and 30 nonobese subjects participated, with 10 to 16 years. There were well overall adiposity by body mass index (BMI), and central adiposity by waist circumference (WC). The cardiorespiratory fitness was assessed by a direct analysis of maximum oxygen consumption $\left(\mathrm{V}_{2 \max }\right.$. Are determined levels of total cholesterol (TC), high density lipoprotein (HDL-C), low density lipoprotein (LDL-C), triglycerides (TG), glucose and insulin after 12 hours of fasting. We analyzed the data by "t" student's test and partial correlation controlled for age, with a significance level of $p<0.05$. The nonobese group had higher values of $V \mathrm{~V}_{2 \max }$ and HDL-C than the obese $(\mathrm{p}<0.001)$. The TG $(\mathrm{p}<0.01)$ and insulin $(\mathrm{p}<$ $0.001)$ were higher in obese compared to non-obese. The LDL-C, TC and glucose didn't differ between groups. There was correlation of $\dot{V} \mathrm{O}_{2 \max }$ was no correlation with $\mathrm{BMI}(\mathrm{r}=-0.50, \mathrm{p}<0.001)$ and the waist abdominal $(r=-0.48, \mathrm{p}$ $<0.001)$, the insulin $(r=-0.392 ; p<0.001)$, TG $(r=-0.277 ; p=0.005)$ and HDL-C $(r=0.299 ; p=0.002)$. There were not found correlations of $V \mathrm{~V}_{2 \max }$ with the TC, the fraction of $\mathrm{LDL}-\mathrm{C}$ and glucose. The lowest $\mathrm{VO}_{2 \max }$ correlated with increased adiposity, insulin and TG, and reduction of HDL However, the $\dot{V} \mathrm{O}_{2 \max }$ not correlated with TC, LDL-C and 
glucose, suggesting the importance of genetic control of these variables and a lower influence of time of inactivity in children and youth population.

UnITERMS: Adiposity; Cardiorespiratory fitness; Lipids; Metabolism.

\section{Referências}

BOUCHARD, C.; DESPRÉS, J. P.; MAURIÈGE, P. O. Genetic and non genetic determinants of regional fat distribution. Endocrinology Review, Baltimore, v.14, p.72-93, 1993.

BOYD, G.S.; KOENIGSBERG, J.; FALKNER, B.; GIDDING, S.; HASSINK, S. Effect of Obesity and High Blood Pressure on Plasma Lipid Levels in Children and Adolescents. Pediatrics, Springfield, v.116, p.442-6, 2005.

CAIOZZO, V.J.; DAVIS, J.A.; ELLIS, J.F.; AZUS, J.L.; VANDAGRIFF, R.; PRIETTO, C.A.; McMASTER, W.C. A comparison of gas exchange induces used to detect the anaerobic threshold. Journal of Applied Physiology: Respiratory Environment Exercise Physiology, Bethesda, v.53, n.5, p.1184-9, 1982

CERCATO, C.; MANCINI, M.C.; ARGUELLO, A.M.C.; PASSOS, V.Q.; VILLARES, S.M.F.; HALPERN, A. Systemic hypertension, diabetes mellitus, and dyslipidemia in relation to body mass index: evaluation of a Brazilian population. Revista do Hospital de Clínicas, São Paulo, v.59, n.3, p.1-10, 2004.

CHU, N.F.; RIMM, E.B.; WANG, D.J.; LIOU, H.S.; SHIEH, S.M. Relationship between anthropometric variables and lipid levels among school children: the Taipei Children Hearth Study. International Journal of Obesity \& Related Metabolic Disorders, Hampshire, v.22, p.66 -72, 1998.

COUTINHO, W. Consenso Latino Americano sobre obesidade 2002. Disponível em: <http://www.abeso.org.br/ consenso.doc>. Acesso em: 8 set. 2006.

DEFORCHE, B.; LEFEVRE, J.; BOURDEAUDHUIJ, I.D.; HILLS, A.P.; DUQUET, W.; BOUCKAERT, J. Physical fitness and physical activity in obese and nonobese Flemish youth. Obesity Research, Baton Rouge, v.11, p.434-41, 2003.

EXPERT COMMITTEE ON THE DIAGNOSIS AND CLASSIFICATION OF DIABETES MELLITUS. Report of the Expert Committee on the Diagnosis and Classification of Diabetes Mellitus. Diabetes Care, New York, v.22, p.s5-s19, 1997. FERNÁNDEZ, J.R.; REDDEN, D.T.; PETROBELLI, A.; ALLISON, D.B. Waist circumference percentiles in nationally representative samples of African-American, European-American, and Mexican-American, children and adolescents. The Journal of Pediatrics, Saint Louis, v.145, p.439-44, 2004.

FREEDMAN, D.S.; KHAN, L.K.; DIETZ, W.H.; SRINIVASAN, S.R.; BERENSON, G.S. Relationship of childhood obesity to coronary heart disease risk factors in adulthood: the Bogalusa Heart Study. Pediatrics, Spfingfield, v.108, n.3, p.712-8, 2001.

FREEDMAN, D.S.; SERDULA, M.K.; SRINIVASAN, S.R.; BERENSON, G.S. Relation of circumferences and skinfold thicknesses to lipid and insulin concentrations in children and adolescents: the Bogalusa Herat Study. American Journal of Clinical Nutrition, Bethesda, v.69, p.308-17, 1999.

GUERRA, S.; DUARTE, J.; MOTA, J. Physical activity and cardiovascular disease risk factors in schoolchildren. European of Physical Education Review, Manchester, v.7, n.3, p.269-81, 2001.

INSTITUTO BRASILEIRO DE GEOGRAFIA E ESTATÍSTICA. Pesquisa de orçamentos familiares 2003-2004. Disponível em: <http://www.ibge.gov.br>. Acesso em: 8 set. 2006.

KIESS, W.; GALLER, A.; REICH, A.; MULLER, G.; KAPELLEN, T.; DEUTSCHER, J.; RAILE, K.; KRATZSCH, J. Clinical aspects of obesity in childhood and adolescence. Obesity Reviews, Oxford, v.2, p.29-36, 2001.

KUCZMARSKI, R.J.; OGDEN, C.L.; GRUMMER-STRAWN, L.M.; FLEGAL, K.M.; GUO, S.S.; WEI, R.; MEI, Z.; CURTIN, L.R.; ROCHE, A.F.; JOHNSON, C.L. CDC growth charts: United States. Hyattsville National Center for Health Statistics, 2000. (Advance data from vital and health statistics of the Centers for Disease Control and Prevention, n.314).

LAKKA, T.A.; LAAKSONEN, D.E.; LAKKA, H-M; MANNIKKO, N.; NISKANEN, L.K.; RAURAMAA, R.; SALONEN, J.T. Sedentary lifestyle, poor cardiorespiratory fitness, and the metabolic syndrome. Medicine Science in Sports Exercise, Madison, v.35, n.8, p.1278-86, 2003.

LEE, S.; BACHA, F.; ARLANIAN, S.A. Waist circumference, blood pressure, and lipid components of the metabolic syndrome. The Journal of Pediatrics, Saint Louis, v.149, p.809-16, 2006.

LEITE, N.; CIESLAK, F; MILANO, G.E.; GÓES, S.M.; LOPES, M.F.A.; BENTO, P.C.B.; PRESTES, A.L.C.; RADOMINSKI, R.B. Associação entre o perfil lipídico e medidas antropométricas indicadoras de adiposidade em adolescentes. Revista Brasileira de Cineantropometria \& Desempenho Humano, Florianópolis, v.11, n.2, p.127-33, 2009 a. 
LEITE, N.; MILANO, G.E.; CIESLAK, F.; LOPES, W.A.; RODACKI, A.; RADOMINSKI, R.B. Effects of physical exercise and nutritional guidance on metabolic syndrome in obese adolescents. Revista Brasileira de Fisioterapia, São Carlos, v.13, n.1, p.73-81, 2009b.

LIMA, S.C.V.C.; ARRAIS, R.F.; ALMEIDA, M.G.; SOUZA, Z.M.; PEDROSA, L.F.C. Perfil lipídico e peroxidação de lipídeos no plasma em crianças e adolescentes com sobrepeso e obesidade. Jornal de Pediatria, Rio de Janeiro, v.80, n.1, p.23-8, 2004.

LISSAU, I.; OVERPECK, M.D.; RUAN, W.J.; DUE, P.; HOLSTEIN, B.E.; HEDIGER, M.L. Body mass index and overweight in adolescents in 13 European Coutries, Israel, and The United States. Archives of Pediatrics \&Adolescent Medicine, Chicago, v.158, p.27-33, 2004.

LOFTIN, M.; SOTHEEN, M.; TRODCLAIR, L.; O'HANLON, A.; MILLER, J.; UDALL, J. Scalin VO2 peak in obese and non-obese girl. Obesity Reviews, Oxford, v.9, p.290-96, 2001.

LOHMAN, T.G.; ROCHE, A.F.; MARTOREL, R. Anthropometrics standartization reference manual. Champaign: Human Kinetics, 1988.

MAFFEIS, C.; SCHENA, F; ZAFFANELLO, M.; ZOCCANTE, L.; SCHULTZ, Y.; PINELLI, L. Maximal aerobic power during running and cycling in obese and non-obese children. Acta Paediatrica, Stockholm, v.83, p.223-6, 1994. MARTINEZ, J.A.; MORENO, M.J.; MARQUEZ-LOPES, I.; MARTÍ, A. Causas de obesidad. Anales del Sistema Sanitariode Navarra, Pamplona, v.25, p.17-27, 2002.

MEHTA, N.K.; CHANG, V.W. Weight status and restaurant availability: a multilevel analysis. American Journal of Preventive Medicine, New York, v.34, n.2, p.127-33, 2008.

OLIVEIRA, C.L.; FISBERG, M. Obesidade na infância e adolescência: uma verdadeira epidemia. Arquivo Brasileiro de Endocrinologia \& Metabologia, São Paulo, v.47, p.107-8, 2003.

ROWLAND, T.W. Exercise and children's health. Champaign: Human Kinetics, 1990.

SAVVA, S.C.; TORNARITIS, M.; SAVVA, M.E.; KOURIDES, Y.; PANAGI, A.; SILIKIOTOU, N.; GEORGIOU, C.; KAFATOS, A. Waist circumference and waist-to-height ratio are better predictors of cardiovascular disease risk factors in children than body mass index. International Journal of Obesity \& Related Metabolic Disorders, Hampshire, v.24, p. 1453-58, 2000.

SHAIBI, G.Q.; CRUZ, M.L.; BALL, G.D.C.; WEIGENSBERG, M.J.; KOBAISSI, H.A.; SALEM, G.J.; GORAN, M.L. Cardiovascular fitness and the metabolic syndrome in overweight Latino youths. Medicine Science in Sports Exercise, Madison, v.37, n.6, p.922-8, 2005.

SOCIEDADE BRASILEIRA DE CARDIOLOGIA. I Diretriz de prevenção da aterosclerose na infância e na adolescência. Arquivos Brasileiros de Cardiologia, São Paulo, v. 85, p.1-36, 2005.

IV Diretriz brasileira sobre dislipidemias e prevenção da aterosclerose. Arquivos Brasileitos de Cardiologia, São Paulo, v.88, p.2-19, 2007.

STENSEL, D.J.; LIN, F.P; HO, T.F; AW, T.C. Serum lipids, serum insulin, plasma fibrinigen and aerobic capacity in obese and nonobese Singaporean boys. International Journal of Obesity \& Related Metabolic Disorders, Hampshire, v.25, p.984-9, 2001.

TANNER, J.M. Growth at adolescence. 2nd ed. Oxford: Blackwell, 1962.

THOMAS, N.E.; COOPER, S.M.; WILLIAMS, S.P.; BAKER, J.S.; DAVIES, B. Relationship of fitness, fatness, and coronary-heart-disease risk factors in 12- to 13-year-olds. Pediatric Exercise Science, Champaign, v.19, p.93-101, 2007. THÖRÖK, K.; SZELÉNYI, Z.; PÔRZÁSZ, I.; MOLNÁR, D. Low physical perfomance in obese adolescent boys with metabolic syndrome. International Journal of Obesity \& Related Metabolic Disorders, Hampshire, v.25, p.966-70, 2001.

YANCEY, A.K.; WOLD, C.M.; McCARTHY, W.J.; WEBER, M.D.; LEE, B.; SIMON, P.A.; FIELDING, J.E. Physical inactivity and overweight among Los Angeles Country adults. American Journal of Preventive Medicine, New York, v.27, n.2, p.146-52, 2004.

ZANCONATO, S.; BARALDI, E.; SANTUZ, P.; RIGON, F.; VIDO, L.; DALT, L.D.; ZACCHELLO, F. Gas exchange during exercise in obese children. European Journal of Pediatrics, Berlin, v.148, p.614-7, 1989.

$$
\begin{array}{r}
\text { ENDEREÇo } \\
\text { Neiva Leite } \\
\text { Núcleo de Pesquisa em Qualidade de Vida } \\
\text { Departamento de Educação Física } \\
\text { Universidade Federal do Paraná } \\
\text { R. Coração de Maria, } 92 \text { - BR 116 - km 95 } \\
\text { 80215-370 - Curitiba - PR - BRASIL } \\
\text { e-mail: neivaleite@gmail.com }
\end{array}
$$

Recebido para publicação: 09/05/2008

Revisado: 21/05/2009

Aceito: 23/07/2009 